\title{
PROPOSTA DE APLICAÇÃO DE RESULTADOS DE SONDAGENS COM MARTELO LEVE (DPL) NO CAMPO EXPERIMENTAL DA UNIFOR EM PROJETOS DE FUNDAÇÕES PARA PEQUENAS CARGAS
}

\author{
Proposal for the Application of Dynamic Probing Light (DPL) Results in the Experimental Field of \\ UNIFOR in Foundations Projects for Small Loads
}

\author{
Marcos Fábio Porto de Aguiar ${ }^{1}$, Fernando Feitosa Monteiro ${ }^{2}$, Francisco Heber Lacerda de Oliveira ${ }^{3}$, \\ Yago Machado Pereira de Matos ${ }^{4}$
}

Recebido em 26 de junho de 2018; aceito em 17 de novembro de 2017; disponível on-line em 26 de julho de 2018.

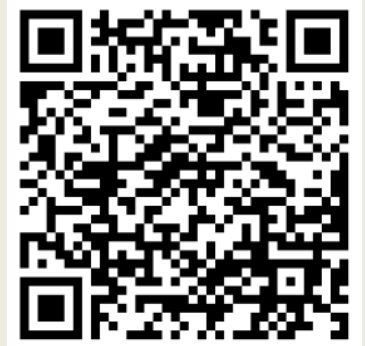

PALAVRAS CHAVE:

Pequenas Edificações;

Tensão Admissível;

Capacidade de Carga;

SPT;

DPL.

\section{KEYWORDS:}

Small Buildings;

Allowable Stress;

Bearing Capacity;

SPT;

DPL.

RESUMO: Em meio aos diversos métodos utilizados no Brasil para determinação da capacidade de carga de fundações, a grande maioria parte do índice de resistência à penetração $\left(\mathrm{N}_{\mathrm{SPT}}\right)$. Para o caso de pequenas edificações, devido a fatores geralmente econômicos, a experiência ou a prática regional costumam prevalecer. Sendo assim, são elaborados, muitas vezes, projetos sem um procedimento de cálculo fundamentado em parâmetros comprovados por ensaios geotécnicos, podendo ocasionar problemas na edificação, como recalques excessivos, ou, até mesmo, comprometer a segurança da estrutura. Dessa forma, técnicas mais simples e de baixo custo, como o DPL (Dynamic Probing Light), podem ser uma opção para situações de pequenas cargas, viabilizando projetos fundamentados em ensaios in situ. Partindo de investigações com o DPL, este trabalho tem o objetivo de dimensionar fundações superficiais e profundas para edificações de pequeno porte através de algumas das principais metodologias disponíveis na literatura e verificar a sua eficiência. Por meio de resultados de campanhas de sondagens SPT (Standard Penetration Test) e DPL no campo experimental da Universidade de Fortaleza (UNIFOR), determinou-se a capacidade de carga de fundações superficiais e profundas fazendo aplicação dos índices $N_{S P T}$ e $N_{S P T}$ equivalente obtido pela correlação com o DPL. Identificou-se que essa correlação apresentou coeficiente de determinação satisfatório entre os parâmetros obtidos nos ensaios SPT e DPL para o terreno em questão, mostrando-se o DPL ser uma alternativa pertinente, em termos técnicos para projetos de fundações de obras de pequeno porte.

ABSTRACT: Among the various methods used in Brazil of the determination of the bearing capacity on foundations, most part uses the standard penetration resistance $\left(\mathrm{N}_{\mathrm{SPT}}\right)$. For small constructions, due to economic factors generally, experience or regional practice usually prevails. Thus, projects without a reasoned calculation procedure in parameters supported by geotechnical tests are often designed, and may cause problems in the building, as excessive settlements, or even compromise the safety of the structure. In this way, simple and low cost techniques such as DPL (Dynamic Probing Light) may be an option for small loads situations, enabling projects based on in situ tests. This paper proposes to calculate the dimensions of shallow and deep foundations for small constructions and check its efficiency using DPL tests. Through the results of SPT (Standard Penetration Test) and DPL tests in the experimental field of the University of Fortaleza (UNIFOR), the determination of the bearing capacity in shallow and deep foundations was done using $N_{S P T}$ index and equivalent $N_{S P T}$ index obtained by the correlation with DPL. A correlation with satisfactory coefficient of determination was obtained between SPT and DPL tests parameters for the analyzed field, showing up the $D P L$ as an appropriate alternative in technical terms for foundation design of small constructions.

* Contato com os autores:

${ }^{1}$ e-mail: marcosfpa@hotmail.com (M. F. P. Aguiar)

Eng. Civil, Doutor, Professor Associado, Instituto Federal do Ceará (IFCE), Fortaleza.

${ }^{2}$ e-mail: engffmonteiro@gmail.com ( F. F. Monteiro )

Eng. Civil, Mestre, Doutorando na Universidade de Brasília (UnB).

${ }^{3}$ e-mail: heberoliveiracivil@hotmail.com ( F. H. L. Oliveira )

Eng. Civil, Doutor, Professor Adjunto, Universidade Federal do Ceará (UFC), Fortaleza.

${ }^{4}$ e-mail: yago_mpm@hotmail.com (Y.M. P. Matos )

Eng. Civil, Mestrando na Universidade Federal do Ceará (UFC). 


\section{INTRODUÇÃO}

É contestável, segundo Bowles (1996), a existência de grandes obras sem o reconhecimento do solo em que estão sendo implantadas. No entanto, para construções menores, há uma prática comum de pouca ou nenhuma investigação. Para Cintra et al. (2013), o solo, diferentemente do concreto e do aço, é um material natural, sem controle para atender às características especificadas, e que, portanto, pode apresentar um comportamento variável quanto à composição e ao comportamento sob carga. Há décadas, partindo inclusive desse conceito de variabilidade do maciço do solo, Terzaghi e Peck (1967) advertiram que um projeto de fundação não pode ser feito de maneira inteligente e satisfatória sem que o projetista tenha, pelo menos, um conhecimento, razoavelmente, preciso das propriedades físicas dos solos envolvidos. Por isso, segundo Cintra et al. (2013), antes de um projeto de fundações, é necessário proceder uma análise do maciço de solo, a chamada investigação geotécnica, com o objetivo de descobrir as condições que a natureza oferece para cada caso particular.

Diante disso, a realização correta de uma obra de engenharia exige um estudo adequado do terreno onde será apoiada a estrutura. Fazendo-se necessário, desse modo, o conhecimento das características da camada de solo subjacente por meio de ensaios de campo e de laboratório, a fim de proporcionar uma maior confiabilidade ao engenheiro durante a elaboração do projeto geotécnico. Sabe-se, no entanto, que, na maioria dos casos, esses procedimentos raramente são adotados, havendo predominância quase que absoluta dos ensaios in situ. Contudo, existem ainda, no Brasil, obras que são construídas sem qualquer investigação geológico-geotécnica do campo, colocando a qualidade e até mesmo a segurança da construção em risco. Nesse cenário, as pequenas edificações predominam, possivelmente, devido às baixas cargas que são transmitidas pela estrutura, resultando em uma menor relevância concedida às fundações. Assim, prescinde-se do conhecimento das características do terreno em busca de um menor dispêndio financeiro, pois os investimentos em sondagens são mais representativos no orçamento final desses empreendimentos. Em meio aos diversos métodos utilizados no Brasil para determinação da capacidade carga de fundações, a grande maioria parte do índice de resistência à penetração ( $\left.\mathrm{N}_{\mathrm{SPT}}\right)$. Para o caso das pequenas edificações, devido a fatores geralmente econômicos, a experiência ou a prática regional costumam prevalecer. Sendo assim, são elaborados, muitas vezes, projetos sem um procedimento de cálculo fundamentado em parâmetros comprovados por ensaios geotécnicos.

Esta prática pode ocasionar problemas na edificação, como recalques excessivos, ou, até mesmo, comprometer a segurança da estrutura. Dessa forma, técnicas mais simples e de baixo custo, como o DPL, podem ser uma opção para situações de pequenas cargas, viabilizando projetos respaldados por ensaios in situ.

\section{OBJETIVO}

O presente trabalho buscou verificar a eficiência do dimensionamento de fundações superficiais e profundas para pequenas edificações por meio dos parâmetros fornecidos por investigações com ensaios DPL. Para isso, além de comparar os resultados obtidos dos dimensionamentos feitos com dados de sondagens SPT, realizou-se, para o caso de fundações profundas, uma retroanálise do dimensionamento por meio de ensaios de prova de carga estática executados em duas estacas do tipo raiz no campo experimental da Universidade de Fortaleza (UNIFOR), seguindo a NBR 12131 que trata de prova de carga estática em estacas (ABNT, 2006).

\section{DYNAMIC PROBING LIGHT (DPL)}

O ensaio de DPL ou Penetrômetro 
Dinâmico Leve, especificado na Referência Internacional para Procedimentos de Ensaio para Sondagem Dinâmica (DP) (ISSMFE, 1989), é, de acordo com Passos (2005), um equipamento manual de pequeno porte, projetado para uso em sondagens in situ com profundidade máxima de 21 $\mathrm{m}$, e, essencialmente, utilizado em solos não coesivos. A Figura 1 ilustra o equipamento e a ponteira cônica.

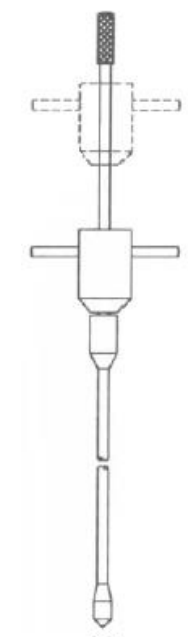

(a)

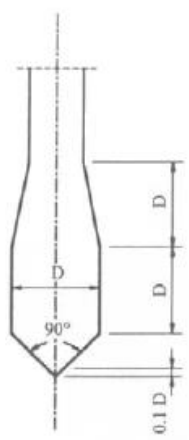

(b)
FIGURA 1: Detalhe [a] equipamento DPL; [b] ponteira. FONTE: Castello et al. (2001).

Mota (2003) afirma que as caraterísticas geométricas e 0 peso do material são especificados pela DIN 4094 (1991), enquanto ISSMFE (1989) define metodologia do ensaio.

Bastante parecido com o SPT, o ensaio de DPL consiste em medir a quantidade de golpes aplicados para cravar uma ponteira em forma de cone por meio da queda livre de um martelo com massa de $10 \mathrm{~kg}$ caindo de uma altura de $50 \mathrm{~cm}$. A resistência à penetração $\left(N_{10}\right)$ é definida como o número de batidas necessário para conduzir o penetrômetro (cone) ao longo de $10 \mathrm{~cm}$. Além do número de golpes, realiza-se, normalmente a cada metro, a medida do atrito lateral da ponteira cônica, que pode ser obtido através do giro do equipamento. Utiliza-se, assim, um torquímetro para medir os torques máximo $\left(\mathrm{M}_{\text {máx }}\right)$ e residual ( $\left.\mathrm{M}_{\text {res }}\right)$ do ensaio.

Como critério de paralisação, Nilsson (2004) sugere que o ensaio deve ser interrompido quando se observar o valor de $\mathrm{N}_{10}>60$ golpes em cinco segmentos sucessivos $(50 \mathrm{~cm})$ ou quando $\mathrm{N}_{10}>80$ golpes em três segmentos consecutivos
(30 cm) ou quando $N_{10}>100$ golpes em um segmento $(10 \mathrm{~cm})$.

Passos (2005) adverte que o fator que limita a cravação do equipamento é a resistência do solo, que estabelece a energia que deve ser aplicada nas hastes. Logo, a energia de um golpe é igual à massa do martelo vezes a aceleração da gravidade multiplicada pela altura de queda (Mgh). Esse fato faz do DPL um ensaio muito adequado para projetos de pequeno e médio porte, servindo de complementação e correlação com outras sondagens. É, portanto, ideal para ser utilizado em locais de difícil acesso, tais como: várzeas, taludes e lugares fechados. Trata-se de um método in situ muito econômico e fácil de usar.

O DPL, segundo Mota (2003), pode ser classificado, quanto à forma de cravação, entre o CPT (estática) e o SPT (dinâmica), pois, apesar de ser um ensaio dinâmico, a energia aplicada é pequena (10 kg com altura de queda de $50 \mathrm{~cm}$ ). Devido a essa baixa energia (aproximadamente 50 J) o ensaio pode ser considerado semiestático. A energia aplicada às hastes, o registro contínuo de resultados (a cada $10 \mathrm{~cm}$ ) e o fato de o elemento de cravação ser uma ponteira cônica tornam o DPL mais correlacionável com o CPT. A determinação da estratigrafia do terreno pode, então, ser feita de forma indireta pelo método proposto por Robertson e Campanella (1983) para o ensaio de cone, relacionando a razão de atrito (FR) com a resistência de ponta do cone (qc) e o tipo de solo, como mostra a Figura 2.

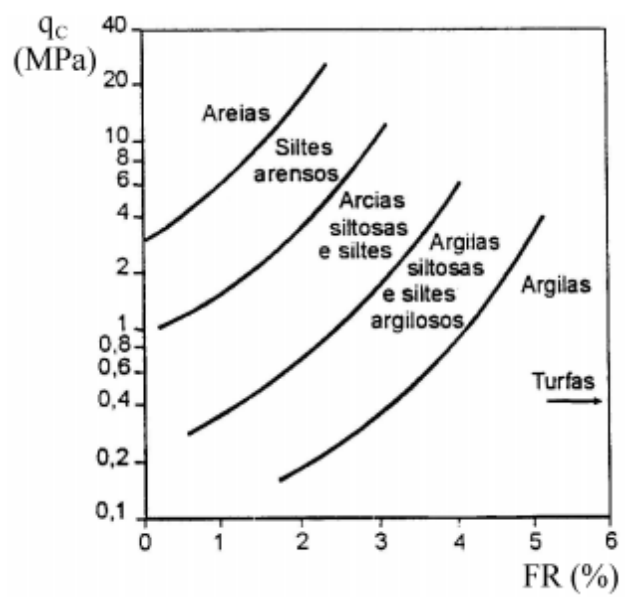

FIGURA 2: Relação entre razão de atrito, resistência de ponta e tipo de solo.

FONTE: Robertson e Campanella (1983). 
A resistência de ponta, no caso do DPL (qd), pode ser obtida através da Equação 1 sugerida por ISSMFE (1989):

$$
q d=\left(\frac{M m}{M m+M v}\right)\left(\frac{M m g h}{A \in}\right) \quad \text { Eq. [1] }
$$

Em que:

$\mathbf{M}_{\mathbf{m}}=$ massa do martelo $(\mathrm{kg})$;

$\mathbf{M}^{\prime}$ = massa total da composição de hastes, cabeça de bater e haste guia $(\mathrm{kg})$;

$\mathbf{h}=$ altura de queda do martelo $(\mathrm{m})$;

$\mathbf{e}=$ penetração média por golpe $(\mathrm{cm})$;

$\mathbf{A}=$ área da base do cone $\left(\mathrm{cm}^{2}\right)$;

$\mathbf{g}=$ aceleração da gravidade $\left(\mathrm{m} / \mathrm{s}^{2}\right)$. pela Equação 2:

0 atrito lateral $\left(f_{s}\right)$ pode ser estimado

$$
f s=\frac{\text { Mres }}{A l}
$$

Sendo:

$\mathbf{A}=$ área de contato do cone com o solo $\left(\mathrm{m}^{2}\right)$;

$\mathrm{I}=$ braço de alavanca do cone $(\mathrm{m})$;

$\mathbf{M}_{\text {res }}=$ momento residual (N.m).

Finalmente, a razão de atrito (FR), medida em porcentagem, é definida na Equação 3 como a relação entre o atrito lateral e a resistência de ponta:

$$
F R=\frac{f s}{q d}
$$

\subsection{CORRELAÇÕES ENTRE $\mathrm{N}_{10}$ E N $\mathrm{N}_{\mathrm{SPT}}$}

Considerando a ampla utilização do SPT para investigar o subsolo e a existência de diversos métodos de dimensionamento de fundações que partem de seus resultados, foram elaboradas algumas proposições que correlacionam os parâmetros obtidos no DPL $\left(\mathrm{N}_{10}\right)$ e no SPT $\left(\mathrm{N}_{\mathrm{SPT}}\right)$.

DIN 1054 (2003) sugere a seguinte expressão (Equação 4) para solos granulares acima do nível d'água quando $3 \leq \mathrm{N}_{10} \leq 50$ :

$$
N s p t=1,4 N 10
$$

DIN 1054 (2003) propõe, também, uma correlação (Equação 5) para solos finos de baixa e média plasticidade acima do nível d'água quando 2 $\leq \mathrm{N}_{10} \leq 30$ :

$$
\text { Nspt }=0,6 \text { N10 }
$$

Acredita-se que, nessas expressões, o valor de $\mathrm{N}_{10}$ se refere à mediana das medidas de cada metro ensaiado.

Vale ressaltar que as correlações fornecidas pela DIN 1054 (2003) não foram concebidas para solos tropicais, típicos do território brasileiro.

No Brasil, alguns autores, partindo da realização de investigações com DPL e SPT, desenvolveram correlações entre os resultados desses ensaios para diferentes localidades.

Entre eles, destaca-se o estudo feito por Nilsson (2004) que apresentou correlações satisfatórias entre os respectivos ensaios para os solos de Curitiba-PR e São José dos Pinhais-PR, predominantemente, de Formação Guabirotuba; e para as argilas porosas de Londrina-PR, CampinasSP e Brasília-DF. Contudo, essas expressões, ainda, são específicas de cada cidade, não sendo recomendadas generalizações para outras regiões.

Sanchez et al. (2010) abordam uma comparação entre diferentes procedimentos de aplicação dos resultados do DPL para obtenção de correlações com o $\mathrm{N}_{\mathrm{SPT}}$. Assim, eles determinaram equações distintas utilizando a soma de $\mathrm{N}_{10}$ nos 30 $\mathrm{cm}$ finais de cada metro e as médias de $\mathrm{N}_{10}$ a cada metro e, também, nos últimos $30 \mathrm{~cm}$ de cada metro. Seus resultados mostraram que a expressão entre o $\mathrm{N}_{\mathrm{SPT}}$ e a média dos valores de $\mathrm{N}_{10}$ obtidos nos últimos $30 \mathrm{~cm}$ de penetração da haste apresentou o maior coeficiente de determinação $\left(R^{2}\right)$ entre as três formas diferentes de correlação.

Porém, o uso de correlações entre os dois ensaios, ainda, parece ser bastante questionável, já que alguns autores não recomendam sua utilização, Silva; Miguel e Belincanta (2006) por exemplo. Outros, como Ribeiro Junior et al. (2007) e Figueiredo (2016), introduziram os resultados do DPL, diretamente, em métodos de cálculo de capacidade de carga de 
estacas.

\section{TENSÃO ADMISSÍVEL DE FUNDAÇÕES SUPERFICIAIS}

No cenário nacional, possivelmente devido ao uso quase exclusivo da sondagem SPT, alguns projetistas empregam apenas os resultados desse ensaio no dimensionamento de fundações superficiais. Algumas correlações consagradas na prática de fundações fornecem, diretamente, o valor da tensão admissível, dispensando, inclusive, fator de segurança, implícito nos cálculos.

Cintra; Aoki e Albiero (2011) ressaltam uma regra para obtenção da tensão admissível de fundações rasas por sapatas, cuja utilização é bastante difundida no meio técnico nacional. A equação considera um valor médio do $\mathrm{N}_{\text {SPT }}$ no bulbo de tensões e uma parcela " $q$ " correspondente à sobrecarga de embutimento da fundação no solo.

É importante mencionar que o bulbo de tensões é a linha imaginária, no interior do terreno, formada por pontos cujos acréscimos de tensão são iguais e que a utilização da sobrecarga "q" no cálculo depende de cada caso, ficando, dessa forma, a critério do projetista. Assim, com 5 $\leq \mathrm{N}_{\text {SPT }} \leq 20$, calcula-se a tensão admissível $\left(\sigma_{\mathrm{a}}\right)$ em kPa conforme a Equação 6:

$$
\sigma a=\frac{N s p t}{50}+q
$$

Para o cálculo da sobrecarga, Cintra; Aoki e Albiero (2011) afirmam que Godoy (1972), partindo de estados de consistência e de compacidade cuja determinação é feita em função do $N_{S P T}$, sugeriu que o peso específico dos solos argilosos e arenosos pudesse ser estimado pelas Quadros 1 e 2 respectivamente.

É válido mencionar que, dificilmente, o valor de $\mathrm{N}_{\mathrm{SPT}}$ é determinado para o primeiro metro de sondagem. Neste caso, seu valor deve ser estimado pelo projetista. Uma alternativa é assumir o valor do primeiro metro como, no máximo, igual ao $\mathrm{N}_{\mathrm{SPT}}$ do metro subsequente.

Outro ponto importante é, se houver oscilação da umidade do solo ao longo da profundidade, utilizar a situação menos favorável, contra a segurança. Para solos argilosos, por exemplo, a pior condição é a saturada.

\begin{tabular}{|c|c|c|}
\hline \multicolumn{3}{|c|}{ QUADRO 1: Peso específico de solos argilosos. } \\
\hline N (golpes) & Consistência & Peso específico $\left(\mathbf{k N} / \mathbf{m}^{\mathbf{3}}\right)$ \\
\hline$<2$ & Muito mole & 13 \\
\hline $3-5$ & Mole & 15 \\
\hline $6-10$ & Média & 17 \\
\hline $11-19$ & Rija & 19 \\
\hline$>40$ & Dura & 21 \\
\hline
\end{tabular}

FONTE: Godoy (1972, apud Cintra, Aoki e Albiero, 2011).

\begin{tabular}{|c|c|c|c|c|}
\hline \multicolumn{5}{|c|}{ QUADRO 2: Peso específico de solos arenosos. } \\
\hline \multirow{2}{*}{ N (golpes) } & Consistência & \multicolumn{3}{c|}{ Peso específico $\left(\mathbf{k} / \mathbf{m}^{\mathbf{3}}\right)$} \\
\cline { 3 - 5 } & Seco & Úmido & Saturado \\
\hline$<5$ & Fofa & 16 & 18 & 19 \\
\hline $5-8$ & Pouco compacta & 17 & 19 & 20 \\
\hline $9-18$ & Medianamente compacta & 17 & 21 \\
\hline $19-40$ & Compacta & 18 & 20 & 219 \\
\hline$>40$ & Muito compacta & 18 & \\
\hline
\end{tabular}

FONTE: Godoy (1972, apud Cintra, Aoki e Albiero, 2011). 


\section{CAPACIDADE DE CARGA DE FUNDAÇÕES PROFUNDAS}

Os métodos semiempíricos se baseiam em resultados de ensaios in situ de penetração (CPT e SPT) e foram ajustados com provas de carga.

Para esses métodos, utiliza-se, geralmente, a equação geral da capacidade de carga originada no equilíbrio entre a carga aplicada, o peso próprio da estaca e a resistência oferecida pelo solo, sendo expressa pela Equação 7:

$$
R=U \sum(r l \Delta l)+\operatorname{rp~Ap}
$$

Em que:

$\mathbf{R}=$ capacidade de carga $(\mathrm{kN})$;

$\mathbf{U}=$ perímetro do fuste da estaca $(\mathrm{m})$;

$\mathbf{A p}=$ área da seção transversal da ponta $\left(\mathrm{m}^{2}\right)$;

$\Delta \mathbf{I}=$ comprimento qualquer da estaca $(\mathrm{m})$;

$\mathbf{r l}=$ resistência lateral $(\mathrm{kPa})$;

$\mathbf{r p}=$ resistência de ponta $(\mathrm{kPa})$.

Cada metodologia possui sua própria consideração sobre as parcelas de resistência lateral e resistência de ponta e, normalmente, adotam coeficientes específicos relacionados com as propriedades do solo do terreno $e$ as características da estaca utilizada. Entre os métodos mais empregados no meio técnico, destacam-se Aoki-Velloso (1975), DécourtQuaresma (1978) e Cabral (1986). Este último é destinado, exclusivamente, a estacas do tipo raiz. Nilsson (2003) desenvolveu uma metodologia para o cálculo da capacidade de carga de fundações profundas por meio de resultados de ensaios DPL que se baseia nas Equações 8, 9 e 10:

$$
\begin{array}{cc}
P=\frac{\left[(q p f A p)+\left(\int_{0}^{Z} f d A s\right)\right]}{F S} & \text { Eq. [8] } \\
q p f=\alpha 1 \alpha 2 q d & \text { Eq. [9] } \\
f=\beta 1 \beta 2 f s & \text { Eq. [10] }
\end{array}
$$

\section{Em que:}

$\mathbf{P}=$ carga de ruptura $(\mathrm{kN})$;

$\mathbf{F S}$ = fator de segurança;

$\mathbf{A p}=$ área da seção transversal da estaca $\left(\mathrm{m}^{2}\right)$;

$\mathbf{d A s}=$ área lateral da estaca $\left(\mathrm{m}^{2}\right)$ correspondente à profundidade $\mathrm{dz}(\mathrm{m})$;

$\mathbf{q p f}=$ resistência de ponta da estaca $(\mathrm{kPa})$;

$\mathbf{f}=$ resistência lateral da estaca $(\mathrm{kPa})$;

qd = resistência de ponta obtida no ensaio DPL $(\mathrm{kPa})$;

$\boldsymbol{\alpha}_{1}=$ fator de correção empírico da resistência de ponta que depende do tipo de solo (Quadro 3);

$\boldsymbol{\alpha}_{2}=$ fator de correção empírico da resistência de ponta que depende do tipo de estaca (Quadro 4); fs = resistência lateral obtida no ensaio DPL $(\mathrm{kPa})$;

$\beta_{1}=$ fator de correção empírico da resistência lateral que depende do tipo de solo (Quadro 3);

$\boldsymbol{\beta}_{2}=$ fator de correção empírico da resistência lateral que depende do tipo de estaca (Quadro 4).

\begin{tabular}{|c|c|c|}
\hline \multicolumn{3}{|c|}{ QUADRO 3: Fatores empíricos de correção para o tipo de solo. } \\
\hline Solo & $\alpha \mathbf{1}$ & $\beta \mathbf{1}$ \\
\hline Curitiba (geral) & 0,2 & 0,6 \\
\hline Curitiba (formação Guabirotuba) & 1,8 & 1,3 \\
\hline Curitiba (material aluvionar grosseiro) & 1,5 & 1,8 \\
\hline Campinas (argila porosa colapsível) & 0,5 & 1 \\
\hline Brasília (argila porosa colapsível) & 0,5 & 1,1 \\
\hline Londrina (argila porosa colapsível) & 0,2 & 0,3 \\
\hline
\end{tabular}

FONTE: Nilsson (2003) 
QUADRO 4: Fatores empíricos de correção para o tipo de estaca.

\begin{tabular}{|c|c|c|}
\hline Estaca & $\mathbf{\alpha 2}$ & $\mathbf{\beta 2}$ \\
\hline Estaca escavada & 0,2 & 1,2 \\
\hline Tubulão encamisado & 0,4 & 0,1 \\
\hline Estaca hélice contínua & 0,2 & 2 \\
\hline Estaca ômega & 0,6 & 2,8 \\
\hline Estaca pré-moldada cravada em argila porosa & 0,5 & 0,7 \\
\hline
\end{tabular}

FONTE: Nilsson (2003).

\section{METODOLOGIA}

Inicialmente, foi feita a remoção de amostras deformadas e indeformadas do campo experimental até uma profundidade de $4 \mathrm{~m}$. Alguns ensaios de laboratório foram realizados, como índices de consistência, granulometria, peso específico dos sólidos, peso específico seco e peso específico natural.

Posteriormente, foram iniciadas as campanhas de sondagem SPT cujos resultados foram usados para o dimensionamento das estacas do tipo raiz.

As estacas raiz foram, então, executadas e levadas até o estado de ruptura por meio de ensaios de prova de carga estática.

Antes da confecção do equipamento DPL, foi feita a pesquisa de resultados de investigações e trabalhos científicos. O DPL foi construído seguindo as caraterísticas geométricas e os pesos de cada componente do equipamento especificados por DIN 4094 (1991). Logo, as campanhas dos ensaios DPL foram realizadas contemplando a metodologia de ensaio definida por ISSMFE (1989). Foram escolhidos, portanto, os procedimentos para correlacionar as medidas de $\mathrm{N}_{\text {SPT }}$ com $\mathrm{N}_{10}$ e determinar o valor índice $\mathrm{N}_{\mathrm{SPT}}$ equivalente.

Após a determinação das cargas correspondentes, foi possível determinar os resultados de cada método de cálculo de capacidade de carga, fazendo análises comparativas e, finalmente, verificando a eficiência da sondagem com martelo leve no dimensionamento de fundações profundas por meio dos resultados das provas de carga estática. Os métodos semiempíricos utilizados foram
Aoki-Velloso (1975) e Cabral (1986) para $\mathrm{N}_{\text {SPT }}$ e $\mathrm{N}_{\text {SPT }}$ equivalente obtido pela correlação com $\mathrm{N}_{10}$; e Nilsson (2003) para resultados de ensaios DPL. Foi considerado, também, o dimensionamento de uma sapata hipotética no campo experimental para analisar a eficiência do ensaio DPL. Neste caso, adotou-se a Equação 6 consagrada na prática de projetos de fundações diretas. Os valores de tensões admissíveis estimados foram confrontados com o resultado de um dimensionamento feito pelo método teórico de Terzaghi (1943) com fator de segurança de 3 .

\section{ANÁLISE DOS RESULTADOS}

\subsection{ENSAIOS DE LABORATÓRIO}

Inicialmente, antes da execução das sondagens no campo, foi feita a retirada de amostras, até uma profundidade de $4 \mathrm{~m}$, para a realização de alguns ensaios de laboratório, como índices de consistência, granulometria, peso específico dos sólidos, peso específico seco e peso específico natural. Alguns resultados encontram-se resumidos na Tabela 1.

A determinação dos índices de consistência do solo indicou um comportamento não plástico para todas as amostras.

Os ensaios de granulometria mostraram que, até a profundidade de $4 \mathrm{~m}$, o material se tratava de uma areia fina com porcentagem significativa em massa variando entre $60 \%$ e $70 \%$ com $16 \%$ a $21 \%$ de grãos menores que $0,075 \mathrm{~mm}$ e $1 \%$ a $3 \%$ de grãos menores que $0,002 \mathrm{~mm}$. Desse modo, segundo o Sistema Unificado de Classificação dos Solos (SUCS), trata-se de um solo SM. 


\begin{tabular}{c|c|c|c}
\hline \multirow{2}{*}{ Profundidade $(\mathbf{m})$} & TABELA 1: Resumo dos resultados dos ensaios caracterização. \\
\cline { 2 - 4 } & $\gamma \mathbf{s}\left(\mathbf{k N} / \mathbf{m}^{\mathbf{3}}\right)$ & $\gamma \mathbf{n}\left(\mathbf{k N} / \mathbf{m}^{\mathbf{3}}\right)$ & $\gamma \mathbf{\gamma d}\left(\mathbf{k N} / \mathbf{m}^{3}\right)$ \\
\hline 1 & 26,2 & 16,4 & 16,2 \\
\hline 2 & 26,3 & 16,4 & 16,1 \\
\hline 3 & 25,9 & 16,2 & 15,8 \\
\hline 4 & 26,0 & 16,3 & 15,9 \\
\hline & & & FONTE: Autoria Própria.
\end{tabular}

\subsection{ENSAIOS DE CAMPO}

As sondagens foram executadas no campo experimental da UNIFOR localizado na capital do Ceará (entre $3^{\circ} 46^{\prime} 6,92^{\prime \prime}$ S e $3^{\circ} 46^{\prime} 7,24^{\prime \prime}$ S e entre $38^{\circ} 28^{\prime} 25,99^{\prime \prime} \mathrm{O}$ e $38^{\circ} 28^{\prime} 26,29^{\prime \prime}$ O). A disposição dos furos de sondagens, Figura 3, foi feita considerando 3 sondagens SPT com profundidade média de $20 \mathrm{~m}$ executadas até o impenetrável conforme NBR 6484 (ABNT, 2001) e 6 ensaios com DPL atingindo, todos, profundidades de $7 \mathrm{~m}$. Os ensaios de torque foram realizados com torquímetros do tipo analógico que abrangiam uma escala de $100 \mathrm{~N} \mathrm{~m}$ a $400 \mathrm{~N} \mathrm{~m}$. A figura apresenta, também, as locações do poço de retirada de amostras e das duas estacas raiz.
Partindo dos resultados obtidos nas sondagens SPT e DPL, determinaram-se, por regressão linear, equações de correlação entre os parâmetros dos dois ensaios. Os valores do número de golpes $\mathrm{N}_{\mathrm{SPT}}$ foram comparados com os valores de $\mathrm{N}_{10}$ (DPL) de quatro formas diferentes:

i. Média entre todos os valores de $\mathrm{N}_{10}$ obtidos em cada metro;

ii. Média entre os valores de $\mathrm{N}_{10}$ obtidos nos últimos $30 \mathrm{~cm}$ de cada metro;

iii. Soma dos valores de $N_{10}$ obtidos na penetração dos $30 \mathrm{~cm}$ na faixa de influência do $\mathrm{N}_{\mathrm{SPT}}$;

iv. Média dos valores de $\mathrm{N}_{10}$ obtidos na penetração dos $30 \mathrm{~cm}$ na faixa de influência do N SPT.

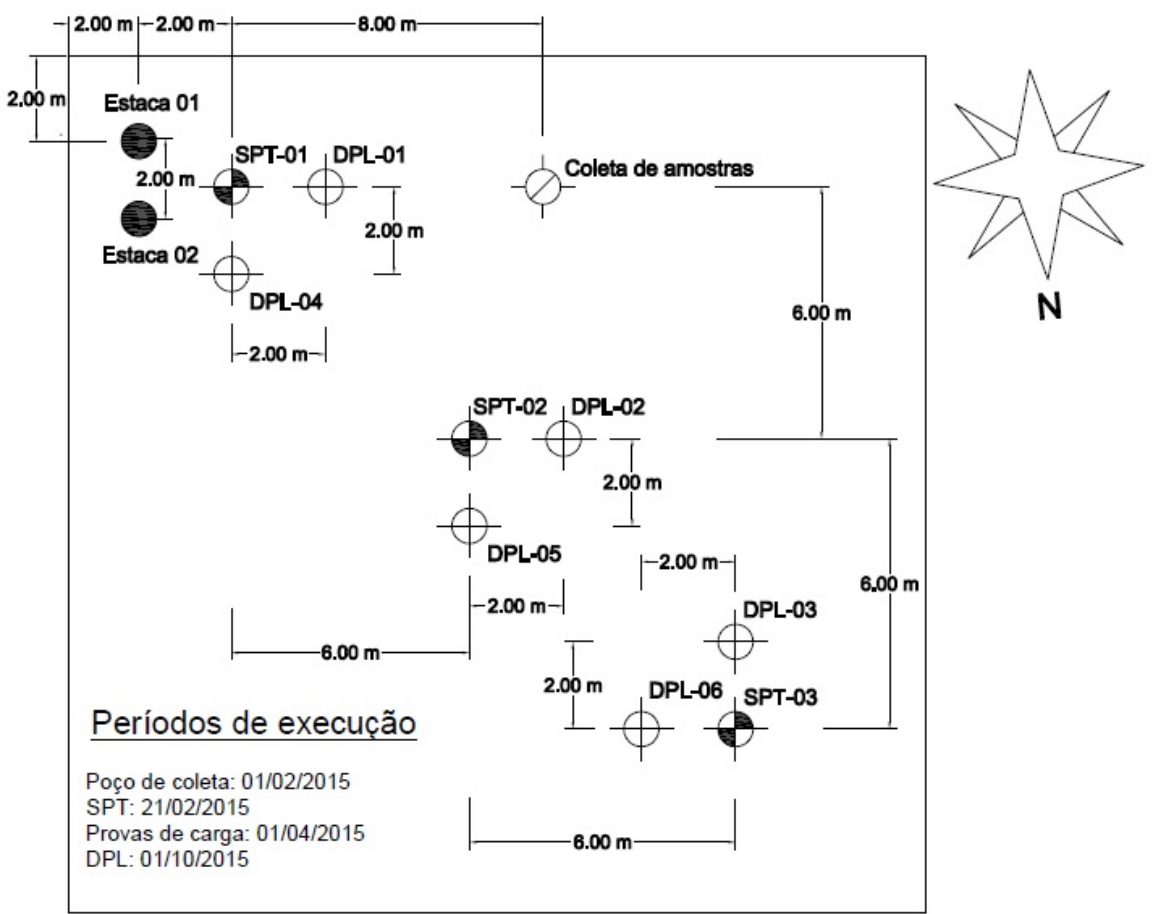

FIGURA 3: Locação dos furos de sondagens SPT e DPL, do poço de coleta de amostras e das estacas raiz no campo experimental da UNIFOR.

FONTE: Autoria Própria. 
A regressão linear foi feita considerando a média de todas as medidas de $\mathrm{N}_{\mathrm{SPT}}$ e a média dos valores de $\mathrm{N}_{10}$ relativos a cada metodologia empregada para cada metro de profundidade, já que não se verificou, de acordo com os desvios padrões calculados, dispersão significativa entre os dados de cada ensaio. Os principais resultados foram:

- Média para cada metro: $\mathrm{N}_{\mathrm{SPT}}=0,5159 \mathrm{~N}_{10}-$ $5,7439\left(R^{2}=0,6578\right)$;

- Média nos últimos $30 \mathrm{~cm}$ de cada metro: $\mathrm{N}_{\mathrm{SPT}}$ $=0,5417 N_{10}-6,1171\left(R^{2}=0,8325\right)$;

- Soma nos $30 \mathrm{~cm}$ da faixa de influência do SPT: $N_{\text {SPT }}=0,2893 N_{10}-7,4776\left(R^{2}=0,9097\right)$;

- Média nos $30 \mathrm{~cm}$ da faixa de influência do SPT: $N_{S P T}=0,5799 N_{10}-7,6208\left(R^{2}=0,9054\right)$.

Portanto, entre todos os conjuntos de dados analisados, a soma e a média dos valores de $N_{10}$ na faixa de influência do ensaio SPT obtiveram os melhores coeficientes de determinação $\left(R^{2}\right)$. Como as duas propostas exibiram, praticamente, o mesmo $R^{2}$, optou-se pela alternativa que possuía os menores valores de coeficiente de variação. A Figura 4 resume a análise realizada.
Dessa forma, foi adotada a média dos valores de $\mathrm{N}_{10}$ na faixa de influência do ensaio SPT. Nesse procedimento, os valores de $\mathrm{N}_{\mathrm{SPT}}$ foram comparados com a média dos golpes $\mathrm{N}_{10}$ na faixa de influência do SPT, ou seja, na cota em que se estima o número de batidas necessário para penetrar os últimos $30 \mathrm{~cm}$ do amostrador. Assim, considerou-se a média dessas medidas para cada metro de sondagem.

Por exemplo, para um ensaio SPT iniciado na cota de $1 \mathrm{~m}$ até a profundidade de 1,45 m, o índice do DPL adotado consiste na média das medidas obtidas nas profundidades de $1,20 \mathrm{~m}$ e $1,30 \mathrm{~m}$, pois, como mencionado anteriormente, o $\mathrm{N}_{10}$ é medido a cada $10 \mathrm{~cm}$.

Vale ressaltar que, devido à existência de variação de estratigrafia do solo no sexto e último metro com relação às camadas sobrejacentes, não foram levadas em consideração as médias dessa profundidade na obtenção da correlação geral, tendo em vista que a intenção do presente trabalho é obter uma expressão específica para o mesmo tipo de solo atravessado. Além disso, a consideração dessa última camada não fornece uma boa correlação entre os dois ensaios. A Tabela 2 esclarece a metodologia adotada.

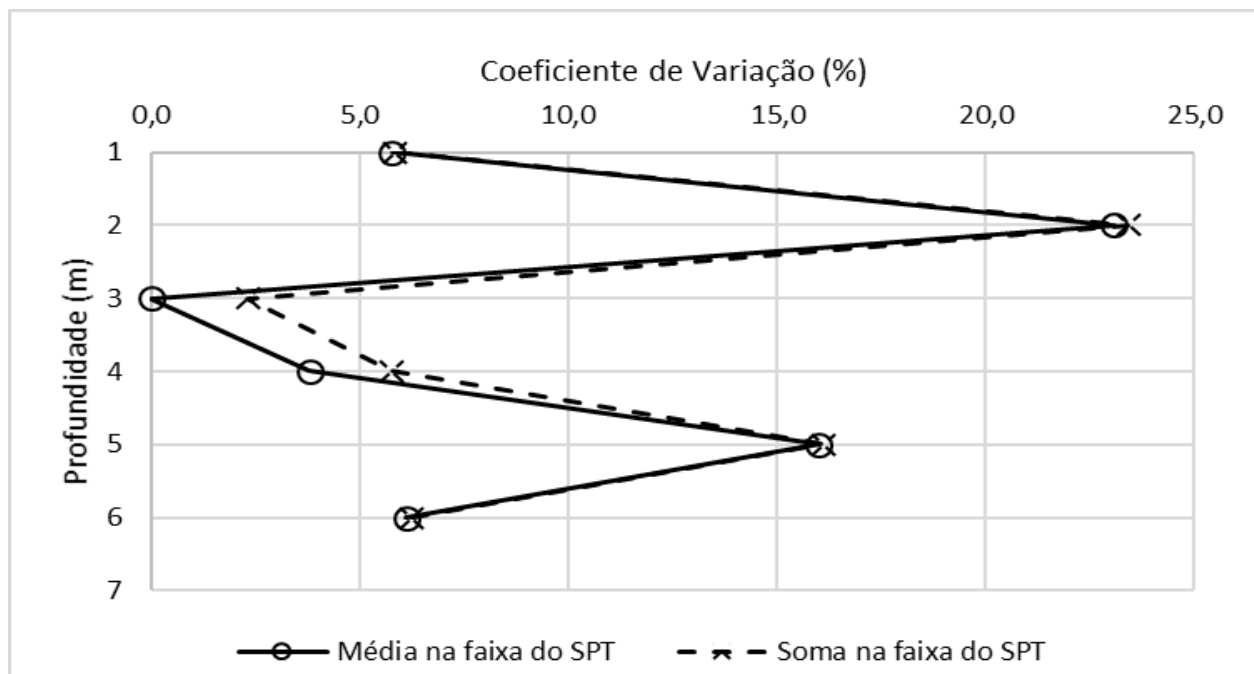

FIGURA 4: Medidas de coeficiente de variação da média e da soma $\mathrm{N}_{10}$ na faixa de influência do SPT ao longo da profundidade.

FONTE: Autoria Própria. 
TABELA 2: Valores de Nspt e N10 considerados na correlação geral

\begin{tabular}{|c|c|c|c|c|c|c|c|c|c|}
\hline \multirow{2}{*}{ Profundidade (m) } & \multicolumn{4}{|c|}{ SPT } & \multicolumn{4}{|c|}{$\mathrm{DPL}$} & \multirow{2}{*}{ Tipo de solo } \\
\hline & Média & $+\sigma$ & $-\sigma$ & $\operatorname{CoV}(\%)$ & Média & $+\sigma$ & $-\sigma$ & $\operatorname{CoV}(\%)$ & \\
\hline 1 & 2 & 2 & 2 & 0 & 17 & 18 & 16 & 6 & Areia siltosa \\
\hline 2 & 2 & 2 & 2 & 0 & 17 & 21 & 13 & 24 & Areia siltosa \\
\hline 3 & 5 & 5 & 5 & 0 & 21 & 21 & 21 & 0 & Areia siltosa \\
\hline 4 & 6 & 8 & 4 & 33 & 26 & 28 & 24 & 8 & Areia siltosa \\
\hline 5 & 8 & 8 & 8 & 0 & 25 & 29 & 21 & 16 & Areia siltosa \\
\hline 6 & 9 & 11 & 7 & 22 & 16 & 17 & 15 & 6 & Silte argilosc \\
\hline
\end{tabular}

FONTE: Autoria Própria.

Com esse conjunto de dados, cuja simplificação fornece a Equação 15 foi definida a equação de correlação geral para areias siltosas do campo experimental da $\left(R^{2}=0,9054\right)$ entre os valores $N_{\text {SPT }}$ e $N_{10}$ (Figura 5), UNIFOR:

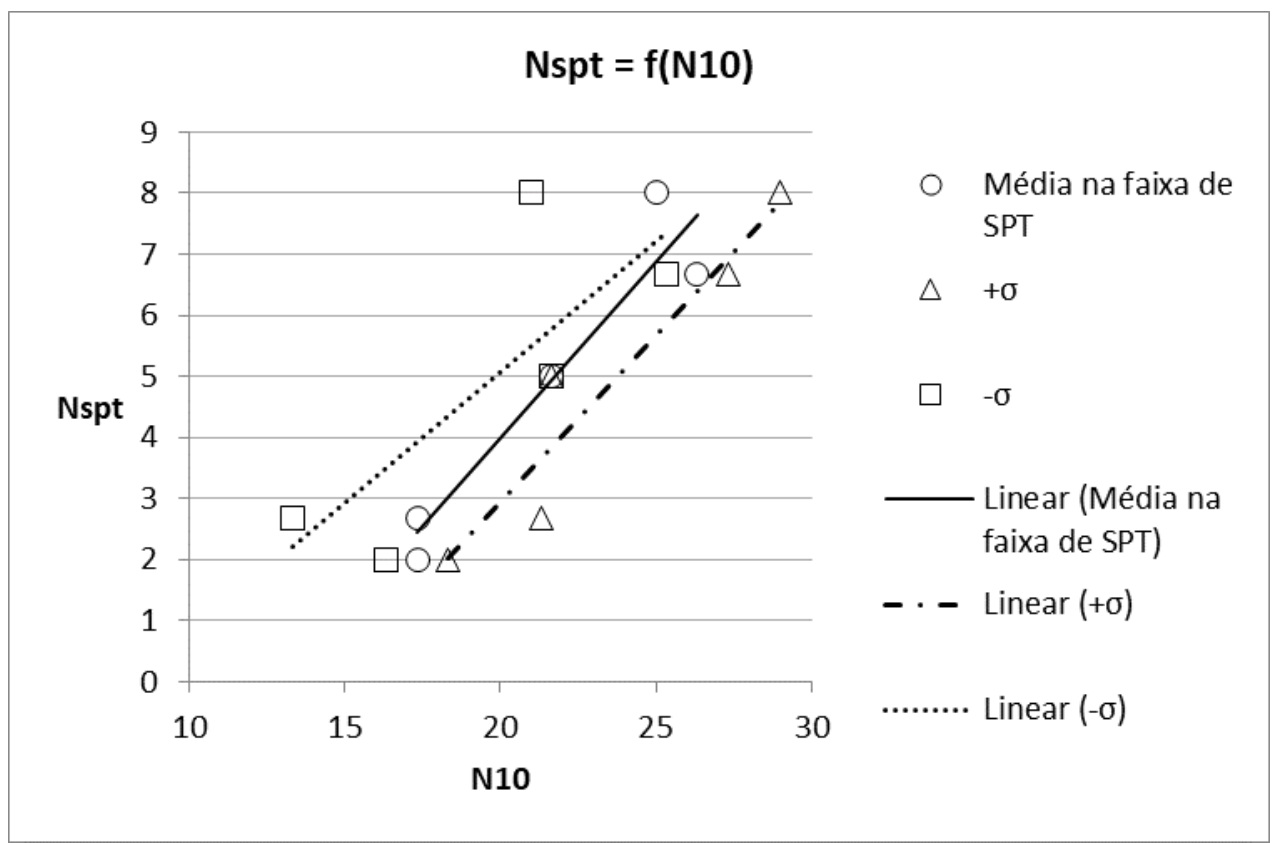

FIGURA 5: Curva de correlação geral.

FONTE: Autoria Própria.

$$
\text { Nspt }=0,6 \mathrm{~N} 10-7,6
$$




\subsection{DETERMINAÇÃO DA TENSÃO ADMISSÍVEL DE UMA SAPATA HIPOTÉTICA}

Para o cálculo da tensão admissível, foi utilizada a Equação 6. Como exemplo de elemento de fundação superficial, adotou-se, no caso, sapata de base quadrada, com 1,5 m de lado e apoiada à profundidade de $2 \mathrm{~m}$.

Considerou-se, para efeitos práticos, a profundidade do bulbo tensões como duas vezes a dimensão da sapata. Utilizou-se, também, a proposta de Godoy (1972) para estimar o peso específico do solo que causa o efeito de sobrecarga na sapata. As tensões admissíveis foram calculadas usando os dados do SPT.

Fazendo uso da correlação geral (Equação 11) obtida entre os resultados das sondagens SPT e DPL no campo experimental da UNIFOR e a Equação 4 sugerida por DIN 1054 (2003) para solos granulares acima do nível d'água foi feita a estimativa do valor do $\mathrm{N}_{\text {SPT. }}$. Em seguida, a determinação da tensão de projeto foi realizada, seguindo a mesma metodologia adotada para o SPT, mas utilizando as camadas de solo reconhecidas pelo ensaio DPL.

A Tabela 3 apresenta a variação de cada um dos valores obtidos em referência aos resultados fornecidos pelo cálculo baseado nos dados do ensaio SPT.

Analisando a Tabela 3, constatou-se que a metodologia de aplicação dos resultados do DPL adotando o $\mathrm{N}_{\text {SPT }}$ equivalente fornecido pela correlação geral apresentou uma aproximação razoável da adotada para o SPT em sapatas, com uma variação máxima entre tensões admissíveis de $15 \%$ menor para o DPL no ponto 02 . No que diz respeito à Equação 4 proposta por DIN 1054 (2003), foram observadas variações bem elevadas (maiores que $300 \%$ ). Possivelmente, por ter sido desenvolvida para solos de países de clima temperado.

A fim de comparar os resultados obtidos do método semiempírico, um dimensionamento foi realizado pela metodologia de Terzaghi (1943) com fatores de capacidade de carga e de forma recomendados por Vesic (1975), adotando-se fator de segurança de 3 e valor médio das sondagens SPT. A Figura 6 ilustra os valores médios de tensão admissível obtido por cada metodologia de cálculo.

\section{TABELA 3: Comparação das tensões admissíveis em sapatas.}

\begin{tabular}{|c|c|c|c|c|c|c|c|c|c|}
\hline \multirow{2}{*}{ Sondagem } & \multirow{2}{*}{ SPT-01 } & \multicolumn{2}{|c|}{ DPL-01 (média) } & \multirow{2}{*}{ SPT-02 } & \multicolumn{2}{|c|}{ DPL-02 (média) } & \multirow{2}{*}{ SPT-03 } & \multicolumn{2}{|c|}{ DPL-03 (média) } \\
\hline & & Correlação geral & DIN (2003) & & Correlação geral & DIN (2003) & & Correlação geral & DIN (2003) \\
\hline Nspt (médio) & 6 & 6 & & 5 & 4 & & 6 & 5 & \\
\hline Tensão admissível (kPa) & 150 & 150 & 710 & 130 & 110 & 650 & 150 & 130 & 610 \\
\hline Variação (\%) & - & 0 & 373 & - & 27 & 333 & - & 13 & 307 \\
\hline
\end{tabular}

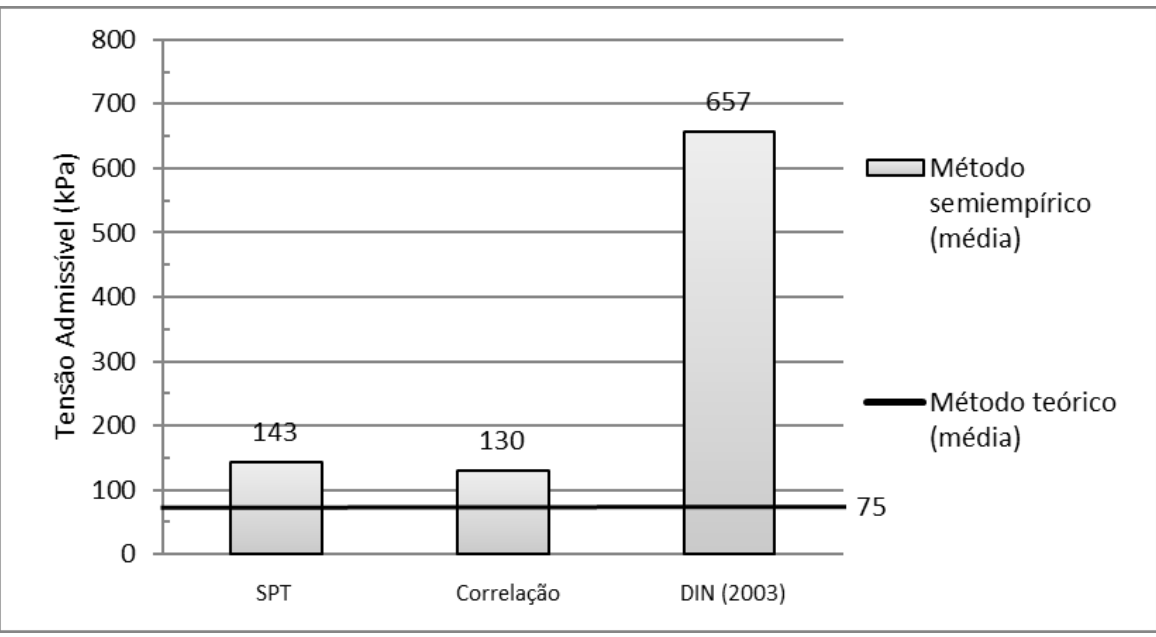

FIGURA 6: Comparação das tensões admissíveis entre os métodos semiempírico e teórico. FONTE: Autoria Própria. 
Observou-se que, para o método semiempírico, o dimensionamento feito com dados do DPL pela correlação geral forneceu resultado próximo do obtido pelo SPT e quase o dobro do valor exibido para o método teórico de Terzaghi (1943).

\subsection{DETERMINAÇÃO DA CAPACIDADE DE CARGA DA ESTACA RAIZ}

\subsubsection{Prova de carga estática}

A execução das duas provas de carga foi feita próxima ao ponto 01 da sondagem SPT com procedimento semelhante ao de carregamento lento conforme a NBR 12131 que trata de prova de carga estática em estacas (ABNT, 2006). As fases de carregamento dos ensaios foram realizadas de acordo com todas as prescrições estabelecidas na referida norma. A princípio, deveria ser feita a aplicação de cargas conhecidas e sucessivas em dez estágios de carregamento, cada um recebendo $10 \%$ do valor da carga de ruptura (estimada em $800 \mathrm{kN}$ ), levando, ao final do ensaio, os elementos de fundação ao estado-limite último. Contudo, em alguns estágios, quando se pretendia obter uma curva carga $\mathrm{x}$ deslocamento mais detalhada ou quando se observava que o valor da carga se aproximava do valor máximo mobilizável pelas estacas, adotaram-se acréscimos de carregamentos inferiores a $10 \%$ da capacidade de carga. As fases de descarregamento das provas de carga não seguiram a NBR 12131 (ABNT, 2006) e foram realizadas em, apenas, um único estágio cada. As Figuras 7 e 8 apresentam os resultados das duas provas de carga executadas no campo experimental.

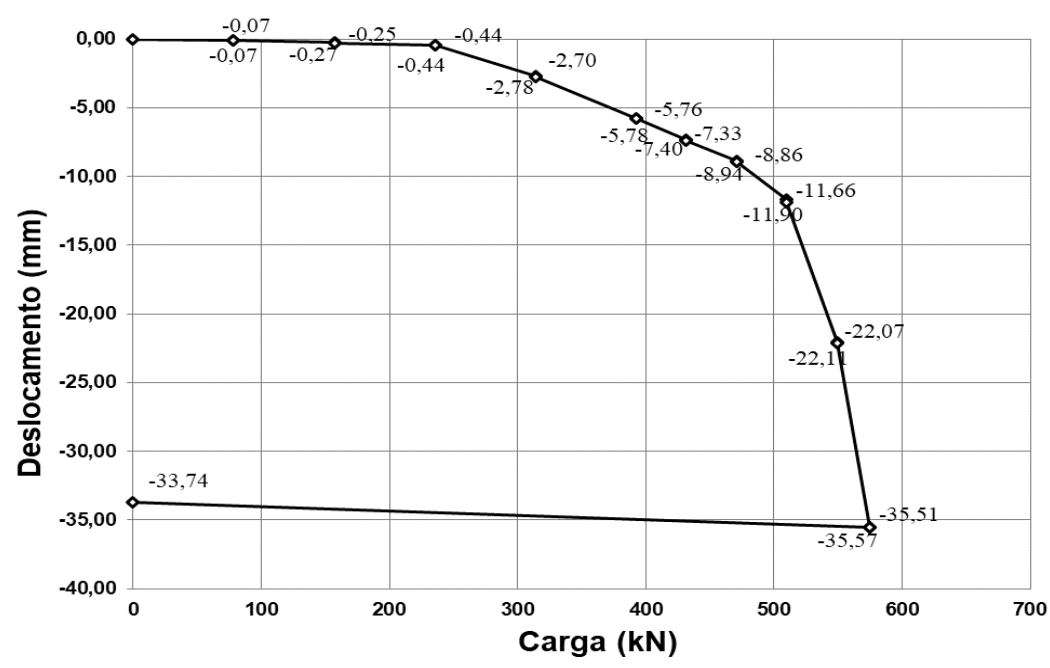

FIGURA 7: Curva carga x deslocamento da estaca E - 01. FONTE: Autoria Própria.

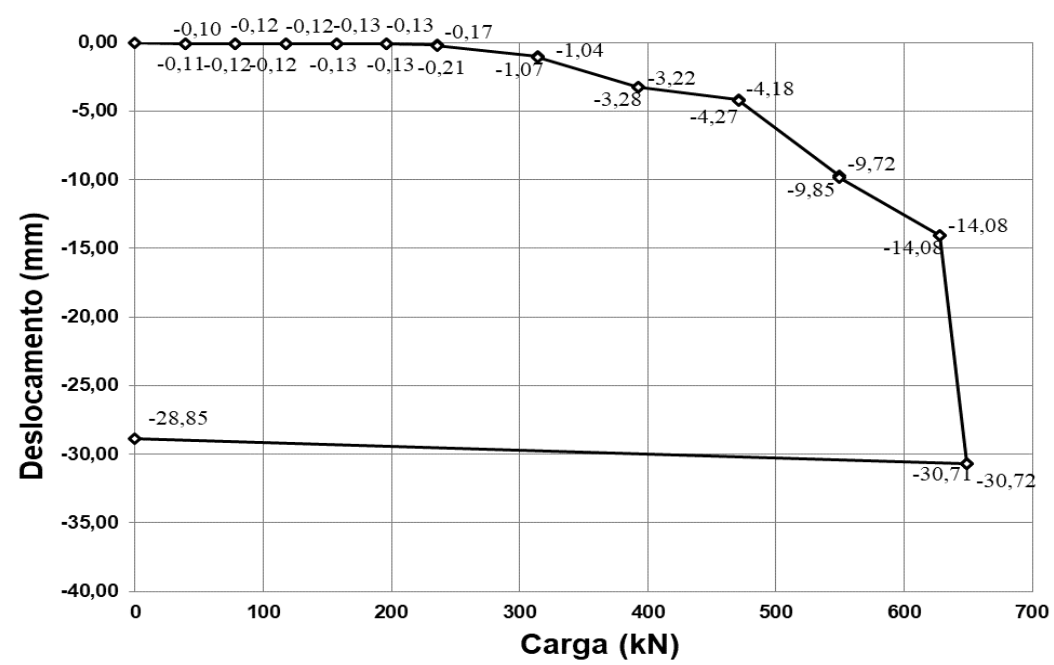

FIGURA 8: Curva carga $x$ deslocamento da estaca $E-02$. FONTE: Autoria Própria. 
Constatou-se que a estaca E - 01 foi submetida a uma carga de ruptura de, aproximadamente, $575 \mathrm{kN}$ e apresentou um recalque máximo de $35,57 \mathrm{~mm}$. A estaca $\mathrm{E}-02$ exibiu um comportamento carga-recalque mais rígido, para uma carga de ruptura em torno de 650 $\mathrm{kN}$, recalcou $30,72 \mathrm{~mm}$.

\subsubsection{Métodos semiempíricos}

Para resultados de ensaios SPT e DPL, o cálculo da capacidade de carga foi feito pelos métodos AokiVelloso (1975) e Cabral (1986). Evidentemente, a proposta de Nilsson (2003) foi aplicada apenas para dados do DPL. Para essa estimativa, utilizaram-se estacas semelhantes às executadas no campo experimental, isto é, tipo raiz com comprimento de $6 \mathrm{~m}, 310 \mathrm{~mm}$ de diâmetro e considerando uma pressão de injeção de $3 \mathrm{kgf} / \mathrm{cm}^{2}$ ( $\approx 294 \mathrm{kPa}$ ). No método de Nilsson (2003), devido à inexistência de dados referentes ao solo de Fortaleza, os fatores de correção empíricos referentes ao tipo de solo foram estimados seguindo as orientações de Nilsson (2003) através de retroanálises das duas provas de carga executadas. Os valores $\alpha_{1}$ e $\beta_{1}$ obtidos para o subsolo do campo experimental da UNIFOR foram 2,1 e 0,7 respectivamente.

No caso do DPL, quanto à estratigrafia do terreno, foram utilizadas as classificações identificadas no referido ensaio, obtidas pelo método proposto por Robertson e Campanella (1983). Assim, após a obtenção do $N_{S P T}$ equivalente pela correlação geral e pela expressão sugerida por DIN 1054 (2003), realizou-se o cálculo da capacidade de carga adotando os mesmos procedimentos empregados para SPT.

As Tabelas 4 e 5 apresentam as cargas de ruptura das estacas por meio das sondagens SPT e DPL além da variação de cada valor, mais uma vez, em relação ao cálculo baseado nos resultados do SPT.

No que diz respeito às fundações por estacas, o procedimento que fornece 0 $\mathrm{N}_{\text {SPT }}$ equivalente pela correlação geral apontou certa variabilidade entre os valores do SPT, 23\%, $54 \%$ e $13 \%$ menores para o DPL usando AokiVelloso (1975) e 24\%, $45 \%$ e $19 \%$, também, menores para o DPL usando Cabral (1986) respectivamente para os pontos 01,02 e 03 . Já a expressão proposta por DIN 1054 (2003) continuou exibindo variações bem elevadas e contra a segurança tanto em Aoki-Velloso (1975) como em Cabral (1986).

Finalmente, a Figura 9 exibe os valores de capacidade de carga obtidos por todos os métodos adotados neste trabalho. Admitindo-se, agora como referência, o menor valor de capacidade de carga entre as duas estacas ensaiadas, ou seja, 575 kN. Foram utilizadas as médias dos resultados de cada método semiempírico.

\begin{tabular}{|c|c|c|c|c|c|c|c|c|c|}
\hline \multirow{2}{*}{ Sondagem } & \multirow{2}{*}{ SPT-01 } & \multicolumn{2}{|c|}{ DPL-01 (média) } & \multirow{2}{*}{ SPT-02 } & \multicolumn{2}{|c|}{ DPL-02 (média) } & \multirow{2}{*}{ SPT-03 } & \multicolumn{2}{|c|}{ DPL-03 (média) } \\
\hline & & Correlação geral & DIN (2003) & & Correlação geral & DIN (2003) & & Correlação geral & DIN (2003) \\
\hline Capacidade de carga (kN) & 497,8 & 382,7 & 1557,4 & 347,6 & 158,9 & 947,6 & 175,8 & 153,3 & 1019,0 \\
\hline Variação (\%) & - & 23 & 213 & - & 54 & 173 & - & 13 & 480 \\
\hline
\end{tabular}

FONTE: Autoria Própria.

\begin{tabular}{|c|c|c|c|c|c|c|c|c|c|}
\hline \multirow{2}{*}{ Sondagem } & \multirow{2}{*}{ SPT-01 } & \multicolumn{2}{|c|}{ DPL-01 (média) } & \multirow{2}{*}{ SPT-02 } & \multicolumn{2}{|c|}{ DPL-02 (média) } & \multirow{2}{*}{ SPT-03 } & \multicolumn{2}{|c|}{ DPL-03 (média) } \\
\hline & & Correlação geral & DIN (2003) & & Correlação geral & DIN (2003) & & Correlação geral & DIN (2003) \\
\hline Capacidade de carga (kN) & 542,0 & 410,9 & 1845,3 & 395,1 & 218,2 & 1394,2 & 287,1 & 233,3 & 1467,1 \\
\hline Variação (\%) & - & 24 & 240 & - & 45 & 253 & - & 19 & 411 \\
\hline
\end{tabular}




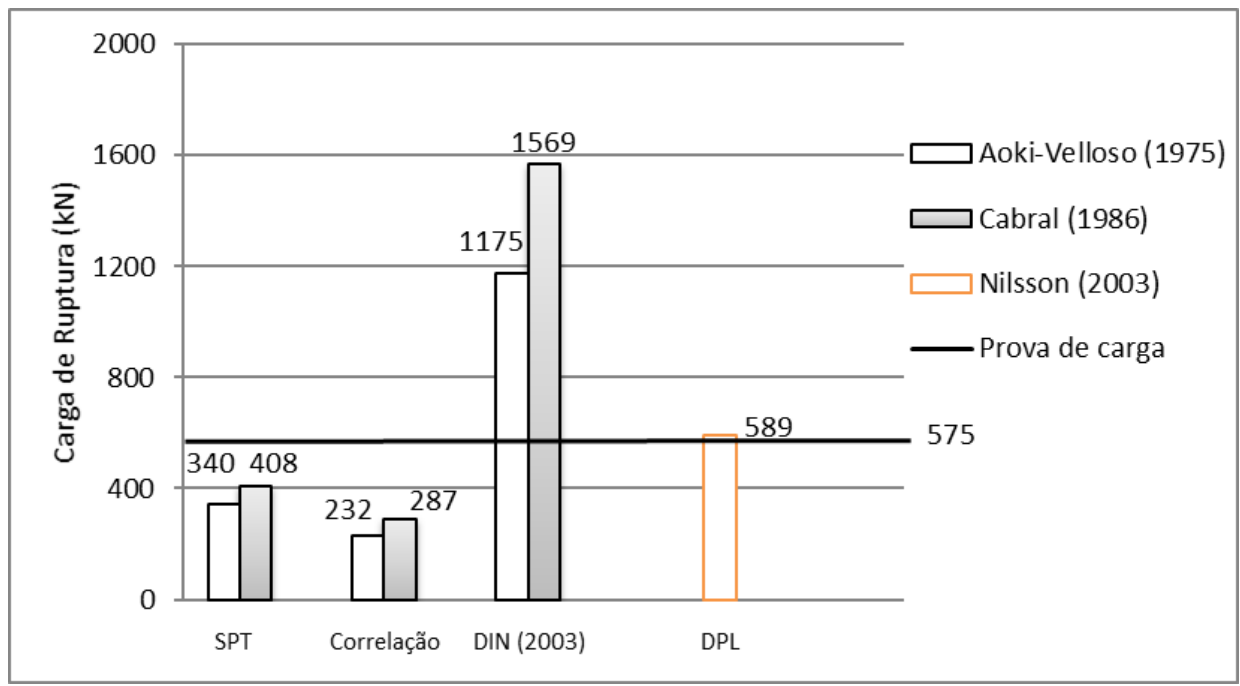

FIGURA 9: Comparação das cargas de ruptura por todas as metodologias empregadas. FONTE: Autoria Própria.

Constatou-se que, entre os três métodos semiempíricos empregados no dimensionamento das estacas, o método de Nilsson (2003) desenvolvido para ensaios DPL se aproximou mais do valor de capacidade de carga, $589 \mathrm{kN}$.

\section{CONCLUSÃO}

O presente estudo possibilitou verificar a viabilidade técnica da utilização de sondagens com o DPL para dimensionamento de fundações superficiais e profundas em projetos de pequenas edificações.

Por meio dos cálculos realizados, constatou-se que a metodologia de aplicação dos resultados do DPL por meio da correlação geral apresentou uma aproximação razoável da adotada para o SPT em sapatas e quase o dobro do valor exibido para o método teórico de Terzaghi (1943). Esses fatos se devem, talvez, à pequena relevância concedida ao tipo de solo no método semiempírico empregado, tendo o número de golpes $\mathrm{N}_{\mathrm{SPT}}$ maior importância para o dimensionamento.

Contudo, no que diz respeito às fundações por estacas, o procedimento apontou certa variabilidade entre os valores do SPT usando tanto Aoki-Velloso (1975) como Cabral (1986), resultando em medidas menores para ambos os métodos com o DPL. Acredita-se que essa irregularidade esteja relacionada, principalmente, com a forma indireta do DPL de determinar a estratigrafia do terreno, a qual foi desenvolvida para solos de clima temperado. Os ensaios DPL apresentaram classificações do solo distintas das obtidas nas sondagens SPT. Portanto, como nos métodos semiempíricos utilizados o tipo de solo atravessado está, diretamente, vinculado com a medida da capacidade de carga das estacas, atribuiu-se essa diferença de valores, sobretudo, à classificação do solo e ao valor do $\mathrm{N}_{\text {SPT }}$. Considerase, também, que os resultados dos ensaios de torque, provavelmente, tenham sido prejudicados pela limitação da escala de medição dos torquímetros operados, o que, para a técnica do DPL, influi, diretamente, na classificação do solo.

O método de Nilsson

(2003) desenvolvido apenas para ensaios DPL forneceu resultados próximos aos encontrados pelas duas provas de carga. Porém, o fato de a estimativa dos fatores de correção empíricos do solo de Fortaleza terem sido obtidos por retroanálise das mesmas duas provas de carga adotadas como referência, provavelmente, influenciou o cálculo da capacidade de carga por essa metodologia.

Observou-se, também, que todas as metodologias baseadas no $\mathrm{N}_{\text {SPT }}$ equivalente proveniente da equação proposta por DIN 1054 (2003), tanto para sapatas como para estacas, revelaram valores bastante discordantes e contra 
a segurança do projeto. Isso pode ser justificado, talvez, pela provável diferença nas propriedades entre o solo usado na obtenção dessa correlação, que é de clima temperado, e o solo do campo experimental, de clima tropical, além de possíveis variações nas eficiências dos ensaios de cada região.

Identificou-se uma correlação com o coeficiente de determinação satisfatório entre os parâmetros obtidos nos ensaios SPT e DPL para o terreno em questão, mostrando-se o DPL ser uma alternativa pertinente, em termos técnicos para projetos de fundações de obras de pequeno porte.

Verificou-se que o dimensionamento por meio da correlação geral do DPL resultou, para essa análise, em valores de capacidade de carga mais conservadores do que os aferidos pela sondagem com o SPT. Essa evidência, se confirmada em outras análises, deve ser levada em consideração para a confirmação da viabilidade econômica do DPL em projetos de fundações para pequenas edificações.

É importante salientar que, apesar de a equação de correlação geral ter apresentado uma boa estimativa do valor NSPT $_{\text {, é necessária cautela }}$ na utilização da referida expressão, pois é importante atentar-se para o fato de ela relacionar dois ensaios que foram realizados em períodos diferentes, cujos dispositivos e metodologias de execução não são equivalentes, provavelmente, com eficiências também distintas e que transpõem um material não homogêneo, o solo. Alternativa viável para definir uma possível equação que correlacione bem os parâmetros $\mathrm{N}_{\text {SPT }}$ e $\mathrm{N}_{10}$ e que seja mais abrangente seria partindo da relação entre energias empregadas em cada um dos ensaios.

\section{AGRADECIMENTOS}

Os coutores $\begin{array}{r}\text { agradecem ao } \\ \text { Desenvolvimento }\end{array}$
Conselho
Científico e Tecnológico (CNPq) e à Fundação
Cearense de Apoio ao Desenvolvimento
Científico e Tecnológico (FUNCAP) pelo apoio
financeiro, ao Instituto Federal de Educação,

Ciência e Tecnologia do Ceará (IFCE), pelo auxílio na confecção do equipamento DPL, à Universidade de Fortaleza (UNIFOR) por ter cedido o campo experimental para realização dos estudos, à Berater Tecnologia de Solos e Fundações pela realização das sondagens SPT e à Tecnord Tecnologia de Solos e Fundações pela execução das estacas e das provas de carga.

\section{REFERÊNCIAS BIBLIOGRÁFICAS}

AOKI, N.; VELLOSO, D. A. An approximate method to estimate the bearing capacity of piles. In: PAN AMERICAN CSMFE, 5., 1975, Buenos Aires. Anais... . Buenos Aires, 1975. p. 367 - 376.

ASSOCIAÇÃO BRASILEIRA DE NORMAS TÉCNICAS. NBR 6484: Solo - Sondagens de simples reconhecimento com SPT - Método de ensaio. Rio de Janeiro: ABNT, 2001.

ASSOCIAÇÃO BRASILEIRA DE NORMAS TÉCNICAS. NBR 12131: Estacas - Prova de carga estática - Métodos de ensaio. Rio de Janeiro: ABNT, 2006.

BOWLES, J. E. Foundation analysis and design. New York: McGraw-Hill, 1996.

CABRAL, D. A. O uso da estaca raiz como fundação de obras normais. In: CBMSEF, 8., 1986, Porto Alegre. Anais... . Porto Alegre, 1986. p. 71 - 82.

CASTELLO, R. R.; POLIDO, U. F.; BICALHO, K. V. Recalques observados de sapatas em solos terciários de São Paulo. Solos e Rochas, São Paulo, v. 24, n. 2, p.143-153, 2001.

CINTRA, J. C. A.; AOKI, N.; ALBIERO, J. H. Fundações diretas: Projeto geotécnico. São Paulo: Oficina de Textos, 2011

CINTRA, J. C. A. et al. Fundações: Ensaios estáticos e dinâmicos. São Paulo: Oficina de Textos, 2013.

DEUTSCHES INSTITUT FÜR NORMUNG. DIN 4094: Erkundung und Untersuchung des Baugrunds. Berlin: Beuth, 1991.

DEUTSCHES INSTITUT FÜR NORMUNG. DIN 1054: Sicherheitsnachweise im Erd- und Grundbau. Berlin: Beuth, 2003.

FIGUEIREDO, L. C. Medida de energia do DPL com instrumentação dinâmica. 2016. 215 f. Tese (Doutorado) - Curso de Geotecnia, Departamento de Engenharia Civil e Ambiental, Universidade de Brasília, Brasília, 2016.

GODOY, N. S. Fundações: Notas de Aula, Curso de Graduação. São Carlos (SP): Escola de Engenharia de São Carlos - USP, 1972. 
INTERNATIONAL SOCIETY FOR SOIL MECHANICS AND FOUNDATIONS ENGINEERING. Report of the ISSMFE technical committee on penetration testing of soils - TC 16 with reference test procedures: International reference test procedure for dynamic probing (DP). Linköping: Swedish Geotechnical Society, 1989. 49 p.

MOTA, N. M. B. Ensaios avançados de campo na argila porosa de Brasília: interpretação e aplicação em projetos de fundação. 2003. 335 f. Tese (Doutorado) Curso de Geotecnia, Departamento de Engenharia Civil e Ambiental, Universidade de Brasília, Brasília, 2003.

NILSSON, T. Initial experiences of DPL NILSSON. In: SIMPÓSIO DE GEOTECNIA DO CENTRO-OESTE, 1., 2003, Cuiabá. Anais... CD-ROM.

NILSSON, T. U. Comparações entre DPL NILSSON e SPT. In: GEOSUL 2004, IV SIMPÓSIO DE PRÁTICA DE ENGENHARIA GEOTÉCNICA DA REGIÃO SUL, 4., 2004, Curitiba. Anais... Curitiba, 2004. p. 61 - 68.

PASSOS, P. G. O. Melhoramento de solos arenosos com estacas de areia e brita. 2005. $141 \mathrm{f}$. Tese (Doutorado) Curso de Geotecnia, Departamento de Engenharia Civil e Ambiental, Universidade de Brasília, Brasília, 2005.

RIBEIRO JUNIOR, I.; SARTO, M. B.; CONCIANI, W. (2007). Estudo comparativo entre as previsões de capacidade de carga para estacas curtas feitas a partir de dados de SPT e DPL. In: III Simpósio Sobre Solos Tropicais e Processos Erosivos. CD. Cuiabá: CEFETMT. 2007.

ROBERTSON, P. K.; CAMPANELLA, R. G. Interpretation of cone penetration tests. Part I: Sand. Canadian Geotechnical Journal. p. 718-733. nov. 1983.

SANCHEZ, P. F.; BOSZCZOWSKI, R. B.; LACERDA, L. A.; LOYOLA, R. D. Estudo da viabilidade do uso do Penetrômetro Dinâmico Leve (DPL) para projetos de fundações de linhas de transmissão em solos do estado do Paraná. In: CONGRESSO BRASILEIRO DE MECÂNICA DOS SOLOS E ENGENHARIA GEOTÉCNICA, 2010, Gramado. Anais... . Gramado, 2010. p. 1 - 8. Disponível em: <http://www.cobramseg2014.com.br/anais/2010/arqui vos/2010.207.pdf>. Acesso em: 14 set. 2016.

SILVA, D. F.; MIGUEL, M. G. ; BELINCANTA, A. Estudo preliminar de estimativa de capacidade de carga de estacas através de ensaios DPL e DPSH em Londrina/PR. In: CONGRESSO BRASILEIRO DE MECÂNICA DOS SOLOS E ENGENHARIA GEOTÉCNICA, 2006, Curitiba. Anais... . Curitiba, 2006. p. 879-884.

TERZAGHI, K. Theoretical soil mechanics. New York: John Wiley and Sons, 1943.

TERZAGHI, K.; PECK, R. B. Soil mechanics in engineering practice. New York: John Wiley and Sons, 1967.
VESIC, A. S. Bearing capacity of shallow foundations. In: WINTERKORN, H. F.; FANG, H. Y. (ed.) Foundation Engineering Handbook. New York: Van Nostrand Reinhold, Cap.3, p. 121-147, 1975. 